\title{
Variations in the Fundamental Plane of massive galaxies with stellar population, morphology and local density
}

\author{
Christina Magoulas ${ }^{1,2}$, Christopher Springob ${ }^{1}$, Rob Proctor ${ }^{3}$, \\ Matthew Colless ${ }^{1}$, D. Heath Jones ${ }^{4}$, Chiaki Kobayashi ${ }^{5}$, \\ Lachlan Campbell ${ }^{6}$, John Lucey $^{7}$ and Jeremy Mould ${ }^{8}$ \\ ${ }^{1} \mathrm{AAO},{ }^{2} \mathrm{U}$. Melbourne, email: c.magoulas@pgrad .unimelb.edu.au, \\ ${ }^{3}$ U. São Paulo, ${ }^{4}$ Monash University, ${ }^{5}$ ANU, ${ }^{6}$ WKU, ${ }^{7}$ Durham University, ${ }^{8}$ Swinburne University
}

We have measured Fundamental Plane (FP) parameters in the 2MASS J, H and K passbands for 10,000 ellipticals, lenticulars and early-type spiral bulges in the 6dF Galaxy Survey (6dFGS) - a spectroscopic survey of the southern sky with $|b|>10^{\circ}$ (Jones et al. 2009). 6dFGS provides a large near-infrared-selected sample of galaxies for accurately determining the NIR FP and investigating the trends in the FP with stellar population, morphology and environment.

In this study the $\mathrm{FP}$, as defined by $\log R_{e}=a \log \sigma_{0}+b \log I_{e}+c$, is modelled by a 3D Gaussian distribution and the best-fit parameters are determined using a robust maximum likelihood method (Magoulas et al. 2012). The best-fit plane is therefore an unbiased determination of the FP, as the censoring effects and correlated uncertainties present in the data are accounted for with this method.

By fitting the FP to galaxies that span a range of environments, we test the universality of the FP and its consistency across galaxies in the field and galaxies in clusters. We find little variation of the 6dFGS FP slopes with global environment (quantified by group richness) or local environment (quantified by surface density). However there is an offset in $r_{0}$ (the $\log R_{e}$-value of the fitted $\mathrm{FP}$ at a fiducial point in the middle of a sample) with environment such that galaxies in the field and low-density regions are on average about 5\% larger than those in groups and higher-density regions (Magoulas et al. 2012). We also classify the morphological mix of galaxies in our sample and find that ellipticals, lenticulars and the bulges of bulge-dominated spirals lie on the same FP and have similar scatter, although the latter are typically $10 \%$ larger at fixed velocity dispersion and surface brightness (Magoulas et al. 2012).

Global stellar population trends within the FP were derived by binning individual galaxy measurements of stellar population parameters (age, metallicity) in FP space. A significant trend in $r_{0}$ is observed, such that younger galaxies are, in general, $25 \%$ larger compared to the oldest galaxies. We find that massive galaxies exhibit clear stellar population trends in FP space: age varies most strongly orthogonal to the FP, while metallicity variation is almost entirely along the intermediate axis of the FP. Remarkably, none of the stellar population parameters vary along the long axis of the plane, which corresponds to luminosity density (Springob et al. 2012).

We interpret the lack of any trend in stellar populations along the long axis of the FP as being the effect of differing dry merger histories on the central luminosity density. We speculate that age drives all the trends in stellar population with residuals about the plane through its correlation with environment, morphology and metallicity.

\section{References}

Jones D. H., et al. 2009, MNRAS, 399, 683

Magoulas C., et al. 2009, MNRAS, 427, 245

Springob C. M., et al. 2012, MNRAS, 420, 2773 